\title{
ANÁLISIS DEL DISCURSO DE MONSERRATH ASTUDILLO
}

\section{ANALYSIS OF MONSERRATH ASTUDILLO'S SPEECH}

JOEL POMA E. ${ }^{1}$

Recepción: 06 de agosto de 2020

Aceptación: 26 de octubre de 2020

\footnotetext{
${ }^{1}$ Pontificia Universidad Católica del Ecuador, Facultad de Ciencias de la Educación, Quito, Ecuador. joelinrck@gmail.com
} 



\section{ANÁLISIS DEL DISCURSO DE MONSERRATH ASTUDILLO}

\section{ANALYSIS OF MONSERRATH ASTUDILLO'S SPEECH}

Joel Poma E.

Palabras clave: características lingüísticas, género, actriz, masculino, femenino Keywords: linguistic characteristics, gender, actress, male, female

\section{RESUMEN}

La sociolingüística es la disciplina que se encarga del estudio de un idioma en relación con la sociedad. Uno de los aspectos que abarca este campo es la diferencia de la lengua de acuerdo al género. Hombres y mujeres tienen una distinta manera de hablar. En efecto, un estereotipo generalizado describe que los hombres tienden a utilizar un vocabulario vernáculo más frecuentemente que las mujeres. Se desarrolló una investigación descriptiva y cualitativa mediante observación no participante que consistió en el análisis del habla de la actriz ecuatoriana Monserrath Astudillo en seis diferentes vídeos desde la plataforma YouTube. En gran parte de la investigación, la teoría coincide con los resultados. Sin embargo, se hicieron interesantes descubrimientos que mostraban que la mencionada actriz posee ciertas características lingüísti- 
cas que no concuerdan con la mayoría de mujeres. En varios vídeos se aprecia cómo Monserrath usa un vocabulario vernáculo espontáneamente y cómo utiliza muchísimo lenguaje corporal, lo cual no es muy común en las mujeres.
También se observa que su discurso es frontal, sin usar modismos para suavizar sus oraciones. Se concluye que las características del trabajo de Astudillo y los guiones que maneja influyen, de alguna manera, en su habla.

\section{ABSTRACT}

Sociolinguistics is the discipline that deals with the study of a language in relation to society. One of the aspects covered in this field is the difference in language according to gender. Men and women have a different way of speaking. In fact, a generalized stereotype describes that men tend to use a vernacular vocabulary more frequently than women. A descriptive and qualitative investigation was carried out through non-participant observation that consisted of the analysis of the speech of the Ecuadorian actress Monserrath Astudillo in six different videos from the YouTube platform. In most part of the research, the theory agrees with the results. However, interesting discoveries were made that showed that the aforementioned actress possesses certain linguistic characteristics that do not agree with most women. Several videos show how Monserrath uses a vernacular vocabulary spontaneously and how uses a lot of body language, which is not very common in women. It is also observed that her speech is extremely frontal without using any idiom to soften her sentences. It is concluded that the characteristics of her work and the scripts that she manages influence somehow her speech.

\section{INTRODUCCIÓN}

Cuando se trata de hablar, las mujeres y los hombres son muy diferentes. No es solo una cuestión de características fisiológicas, sino del léxico y la forma en que las personas utilizan el lenguaje.
En la sociedad en la que se circunscribe esta investigación, los hombres generalmente hablan usando términos vernáculos para demostrar machismo frente a otros hombres o superioridad hacia 
las mujeres, quienes, por otro lado, son las personas responsables de los valores de la sociedad. Ellas mantienen el equilibrio del idioma al hablar de una manera más civilizada. Martin (1996) afirma que, en el nivel fonológico, se ha señalado en las mujeres un comportamiento más conservador y apegado a la norma (p. 6). Lo que significa que su habla es menos vernácula y se caracteriza por ser más educada. Vernáculo se refiere a "un dicho especialmente del idioma o lengua: doméstico, nativo, de la casa o país propios" (Real Academia Española, s.f., definición 1). Con respecto al vocabulario, Lakoff (1982) asegura que:

Existen tipos similares de disparidades en otras partes del vocabulario. Hay, por ejemplo, un grupo de adjetivos que tienen, además de sus significados específicos y literales, otro uso, el de indicar la aprobación o admiración del hablante para algo. Algunos de estos adjetivos son neutrales en cuanto al sexo del hablante: hombres o mujeres pueden usarlos. Pero otro conjunto parece, en su uso figurativo, ser confinado en gran medida al discurso de las mujeres (p. 51).

Dicho de otra manera, las mujeres emplean palabras más sofisticadas para describir su entorno, al mismo tiempo, procuran ser precisas en su explica- ción utilizando palabras que se acerquen lo máximo al objetivo lingüístico del hablante. Otro de los aspectos enfatizados por este autor es que las mujeres recurren, a menudo, a preguntas eco ("¿no te parece?", "¿verdad?", "¿no?", "¿eh?") con las que tratan de asegurarse de que cuentan con la aprobación de su interlocutor, evitando el conflicto.

Martin (1996) menciona una interesante característica sobre las mujeres: ellas recurren frecuentemente a las respuestas mínimas ("sí, sí", "claro, claro", etc.), las cuales representan una manera de asegurar a su oyente que se le está prestando atención, mientras que los varones las emplean, generalmente, para manifestar que están de acuerdo. Sobre estas características, la autora aclara que:

Todos estos rasgos deben ser considerados como elementos lingüísticos que conformarían el estereotipo de habla femenina, antes que como marcadores de género o elementos que definen a un sociolecto femenino. Estereotipo que responde también a cómo se enseña a hablar a las mujeres, el cual negaría a la mujer la posibilidad de expresarse con fuerza y rotundidad, y favorecería una expresión ligada a la trivialidad y a la falta de criterio propio. Las mujeres pueden adherirse en mayor o menor grado a este estereotipo y la sola 
presencia de alguno de estos rasgos puede servir para evocarlo (p. 7).

Moreno (1998) afirma que a esta situación de actuación lingüística se le conoce también con el nombre de 'variedad basada en el género', en el entendido de que las mujeres tienen habilidades verbales mayores y mejores que los hombres y que, probablemente, pueden ir más allá de las diferencias socioculturales. Se precisa tomar en cuenta cada uno de los puntos lingüísticos antecedidos porque definen teóricamente la manera de hablar de las mujeres y permiten una comparación con las expresiones orales de la actriz Astudillo; por lo tanto, exponer las características principales del habla femenina para cotejarlas con los actos de comunicación en situaciones reales. En esta investigación se analiza por observación seis vídeos sobre la forma de hablar de Monserrath Astudillo, una popular actriz ecuatoriana quien ha participado ampliamente en producciones de radio, televisión y teatro. Los parámetros estudiados son elementos paralingüísticos, sintaxis, vocabulario, organización de temas, estrategia de conversación y lenguaje corporal. El objetivo de este trabajo es determinar si la teoría sobre cómo hablan las mujeres se encuentra en el discurso de esta artista y descubrir cómo pueden variar algunas características. Dado que la comunicación oral de una persona puede diferir dependiendo de diferentes factores intrínsecos y extrínsecos, es importante observar al menos - seis vídeos donde la actriz se desempeña en diversos escenarios, como: laboral, familiar, mediático y social. La elección de una persona reconocida en lugar de una anónima resulta esencial para que este estudio sea más fácil de entender y más rápido de identificar para los lectores. Los resultados, las conclusiones y algunos hallazgos interesantes de la investigación se encuentran al final de este artículo.

\section{MÉTODO DE RECOLECCIÓN DE INFORMACIÓN}

Se seleccionaron los vídeos sobre la base de los parámetros expuestos: los actos de habla de la actriz Astudillo que reflejen los escenarios laboral, familiar, mediático y social.

Cada vídeo fue analizado por me- dio de la observación. Las notas se organizaron según las características a las que pertenecían, tales como: elementos paralingüísticos, sintaxis, vocabulario, organización de temas, estrategia de conversación y lenguaje corporal. La or- 
ganización sistémica contó con matrices para registrar los tiempos (minutos y/o segundos) donde se evidenciaron las características en análisis. Los resultados se compararon con la teoría sobre el discurso de las mujeres. Luego se redactó la conclusión en párrafos cortos después de cada característica para describir si el artista los cumplió o no. Finalmente, los hallazgos descubiertos se describen después de los resultados.

\section{VÍDEOS ANALIZADOS}

1. Monserrath Astudillo: La prueba de amor (2018)

2. Entrevista con la Monse (2015)

3. Tejuro que es mi primera vez Monólogo HD 01.. Teatro Lalama (2017)

4. El Cadáver de mi ex Monserrath AstudiIlo (2020)

5. Monserrath Astudillo - En el baño (2015)

6. Testimonio de Superación-Monserrath Astudillo - Luna y Nuna (2017)

\section{RESULTADOS}

Después del análisis de estos seis vídeos se obtuvieron los siguientes resultados.

\section{Elementos Paralingüísticos}

Entonación enfática: Monserrath usa entonación enfática cuando habla, especialmente cuando está realizando una obra de teatro.

Muestra:

Vídeo 1 - 0:48 "Bueno, ¿virgen? ¿Virgen?"

Extensión de las vocales: la actriz extiende las vocales muy a menudo, en todos los vídeos.

Muestras: nistas"
Vídeo 4 - 0:21 "Cuando una relación se termina"

$$
\begin{aligned}
& \text { Vídeo } 6 \text { - 2:35 "Miedo" } \\
& \text { Vídeo } 6 \text { - 2:54 "Nadie" }
\end{aligned}
$$

\section{Entonación Aumentada-dismi-}

nuida: la artista es una persona que cambia mucho su entonación en cada vídeo.

Muestras:

Vídeo 1 - 4:41 "...romances, quizá.

Pónganse que esté así borracha..."

Vídeo 3 - 0:55 "Todo, todo, todo"

Vídeo 4 - 0:30 "¿Por qué conmigo nunca ha salido en redes sociales?"

Vídeo 5 - 1:08 "Y cuando estas a punto de entrar, llega la viejita"

Vídeo 6 - 3:48 "Esto nos pasa, y en verdad, acoliten"

Vídeo 6 - 4:30 "O sea, en el fondo si" 
Cambio de voz: la actriz cambia la voz debido a su trabajo, pero también en condiciones naturales.

Muestras:

Vídeo 1 - 2:28 "iLa gente!"

Vídeo 4 - 0:54 "iEl longo es influen-

cer!"

Vídeo 5 - 1:14 "Yo voy a entrar primero"

Vídeo 6 - 4:08 ";Qué bestia! ¡Cómo me hace reír!"

Entonación aguda: la voz de la actriz alcanza una entonación aguda cuando quiere enfatizar un punto específico.

Muestra:

Vídeo 3 - 0:57 "¡Todo! jTodo! ¡Todo!"

\section{Sintaxis}

La artista habla en segunda persona.

Muestra:

Vídeo 1 - 2:57 "¿Sí o no chicas?"

Monserrath hace preguntas variadas y usa exclamaciones interrogativas en su discurso.

Muestras:

Vídeo 1 - 0:48 "Bueno, ¿Virgen? ¿Virgen?"

Vídeo 2 - 1:40 "¿Carla o Carlita te gusta que te digan?"

Vídeo 4 - 0:23 "¿Saben qué hacemos?"
Vídeo 5 - 2:35 "¿Dónde pones la cartera si no hay gancho?"

Vídeo 6 - 0:30 "¿Qué pasa si no tengo un hijo y luego me arrepiento de no tener un hijo?"

\section{Vocabulario}

Ella se refiere a temas privados y personales.

Muestras:

Vídeo 1 - 0:23 "Hoy no me peiné..."

Vídeo 6 - 1:50 "No me gustaba estar con mi hijo"

Ella usa diminutivos, pero no demasiados.

Muestras:

Vídeo 4 - 0:13 "Le pones la velita"

Vídeo 5 - 5:45 "Sacudidita"

La actriz cambia los temas cuando habla; incluye diferentes aspectos al hablar sobre un tema. También habla sobre sus propias experiencias y de manera muy personalizada. Se puede observar que estas características aparecen con más frecuencia en los vídeos uno y seis, porque se trata de entrevistas personales. También utiliza algo de estilo íntimo en su discurso.

Muestra:

Vídeo 1 - 3:41 "¿Saben qué me pongo en la cara?" 


\section{Estrategia conversacional}

Se puede observar un ejemplo de cooperación: dos o más mujeres coincidiendo sobre el mismo tema, en el primer vídeo.

Muestra:

Vídeo 1 - 06:24 "Exacto"

\section{Lenguaje corporal}

Ella se acerca mientras habla.

Muestra:
Vídeo 2 - 1:40 (se acerca a la entrevistadora para hacerle una pregunta)

Ella también cruza las piernas en el primer y último vídeo.

Muestras:

Vídeo 1 - 4:17 (cruza las piernas mientras se acomoda)

Vídeo 6 (cruza las piernas constantemente durante toda la entrevista)

\section{HALLAZGOS}

Se presentan algunos hallazgos interesantes. Estas características son las que no siguen la teoría del discurso de las mujeres.

\section{a. Lenguaje Indirecto}

Contrariamente a la mayoría de las mujeres, Monserrath no usa un lenguaje indirecto, sino muy directo para expresarse. Esto se evidencia en todos sus vídeos utilizados para este estudio.

\section{b. Uso de modismos y palabras para minimizar el impacto}

La artista no usa modales u otras palabras para minimizar el impacto de sus frases. Sin embargo, ella agrega palabras comúnmente denominadas "sucias" en su discurso que, aunque podrían resultar incómodas para muchas personas, facilitan la comprensión. Es curioso porque la teoría expuesta en esta investigación advierte que las mujeres generalmente usan modales y ciertas palabras para minimizar el impacto, pero esta persona sale de esa norma.

\section{Muestras:}

Vídeo 1 - 0:41 ".. teta parada..."

Vídeo 1 - 2:47 "...te puntean..."

Vídeo 1 - 3:55 "...Culinovios"

Vídeo 1 - 4:32 ".. hablar huevadas"

Vídeo 1 - 5:42 "...conchas de su madre"

Vídeo 1 - 7:00 "Si usted tira..."

Vídeo 1 - 07:47 "...yo salgo nomás Ilucha"

Vídeo 3 - 0:27 "... me coge el culo" Vídeo 5 - 0:23 "... Brad Pitt en bolas"

Vídeo 5 - 2:13 "Vieja de mierda" 


\section{c. Gestos Faciales}

Otro hallazgo importante es que muchos gestos faciales no son una característica común en una mujer. Sin embargo, esta persona utiliza muchos gestos faciales en su trabajo artístico, especialmente en sus actuaciones (en menor medida en las entrevistas). Además, aunque la teoría manifiesta que las mujeres mantienen sus manos cerca de su cuerpo, Astudillo mueve sus manos ampliamente y lejos de su cuerpo.

Muestras:

Vídeo 1 - 2:03 (muchas expresio- nes faciales y movimiento de sus manos de acuerdo a su habla)

Vídeo 4 - 0:10 (moviendo mucho las manos)

Vídeo 5 - 0:05 (moviendo las manos)

Finalmente, no es tan común observar a una mujer exagerando el lenguaje corporal, y podemos constatar que esta actriz incluso modela frente a la cámara.

Muestra:

Vídeo 1 - 3:15 (Modelando)

\section{CONCLUSIONES}

El carácter performativo del discurso de Monserrat Astudillo materializa formas ajenas, diferentes y poco convencionales a los actos del habla de la mayoría de mujeres ecuatorianas, escenario donde se enmarca el análisis de esta investigación. Debido a su trabajo como actriz, se torna superlativa al momento de crear humor negro de índole cotidiano. Se permite ciertas licencias lingüísticas, por ejemplo, la utilización de malas palabras, y muchas expresiones faciales y coloquiales habituales en su trabajo actoral, pero ajenas a la socialización cultural y los discursos hegemónicos que circulan en la realidad fáctica.
Estas características son parte de su actuación, donde combina su discurso natural con una imitación muy exagerada de la realidad, hecho que queda plasmado principalmente en los vídeos satíricos. El humor negro y hasta el sarcasmo son minimizados cuando es entrevistada sobre temas personales o de otra índole.

Está claro, entonces, que mientras la mayoría de mujeres hablan tratando de suavizar sus expresiones, utilizando un vocabulario más sofisticado y conservador, manteniendo la postura sin caer en la exageración de su lenguaje corporal; el discurso de Astudillo, 
analizado en este estudio, tiene ciertas características que salen del contexto cultural común de las mujeres. Esta investigación analizó la teoría vigente sobre actos de comunicación verbales y la comparó con los hechos observables en el discurso de una actriz reconocida en los escenarios artísticos del país. Por lo tanto, se concluyó que Mon- serrath expresa un discurso contra hegemónico inherente a una minoría de mujeres, que, en términos cuantitativos no representa la praxis comunicacional del entorno femenino, pero que ha encontrado en el quebrantamiento del lenguaje la herramienta fundamental para la deconstrucción de las relaciones sociales interpersonales. 


\section{REFERENCIAS}

Astudillo, M. [Monserrath Astudillo]. (2018, March 21). Moserrath Astudillo: La prueba de amor [Vídeo]. YouTube. https://www.youtube. $\mathrm{com} /$ watch?v=078 LICoJlfk

Astudillo, M. [Monserrath Astudillo]. (2020, June 8). El Cadáver de mi ex, Moserrath Astudillo [Vídeo]. YouTube. https://www.youtube. $\mathrm{com} /$ watch?v=nqqEYZ9XrwA

Galarza, A. [Galaproducciones Aby Galarza]. (2017, May 16). Te juro que es mi primera vez Monólogo HD $01 .$. Teatro Lalama [Vídeo]. YouTube. https://www.youtube.com/watch?v=CPT99EnsuUw

Gamavisión. [Gamavisión]. (2015, June 4). Entrevista con la Monse [Vídeo]. YouTube. https://www.youtube. $\mathrm{com} /$ watch? $\mathrm{v}=\mathrm{oEKAdyJOVpo}$

Gamavisión. [Gamavisión]. (2015, June 18). Monserrath Astudillo - En el baño [Vídeo]. YouTube. https:// www.youtube.com/watch?v=cvCHlihtSes

Lakoff, R. (1982). Language and woman's place. Language in Society. 2(1) https://web.stanford.edu/class/ linguist156/Lakoff_1973.pdf
Luna y Nuna. [Luna y Nuna]. (2017, April 11). Testimonio de Superación - Monserrath Astudillo - Luna y Nuna [Vídeo]. YouTube. https:// www.youtube.com/watch? $v=\mathrm{cv}$ CHlihtSes

Martín, L., Gómez, C., Santamarina, C. (1996) Lenguaje y Género. Descripción y explicación de la diferencia. Quadernsdigitals. http:// www.quadernsdigitals.net/ datos_web/hemeroteca/r_3/ nr_44/a_649/649.html

Moreno, Francisco. (1998) Principios de sociolingüística y sociología del lenguaje. Research Gate. https://www.researchgate.net/ publication/44357930_Principios_de_sociolinguistica_y_sociologia_del_lenguaje_Francisco_Moreno_Fernandez/ link/58ed1192aca2724f0a26b 383/download

Real Academia Española. (s.f.). Vernáculo. En Diccionario de la lengua española. Recuperado en 15 de octubre de 2020, de https://dle.rae.es/ vern\%C3\%A1culo 